\title{
Avispas parasitoides Ophioninae (Hymenoptera: Ichneumonidae) del departamento de Arequipa, Perú
}

\author{
Parasitoid wasps Ophioninae (Hymenoptera: Ichneumonidae) from Arequipa Department,
} Perú

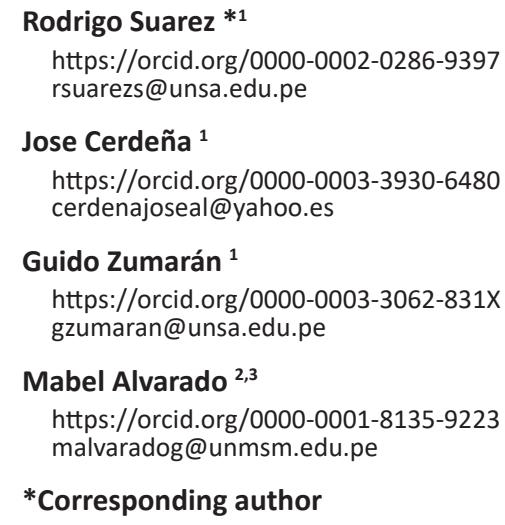

1 Universidad Nacional de San Agustín de Arequipa, Calle Santa Catalina 117, Arequipa, Perú.

2 Universidad Nacional Mayor de San Marcos, Museo de Historia Natural, Departamento de Entomología, Lima, Perú.

3 Bosque Llaqta, Av. Confraternidad Internacional, Perú.

\section{Citación}

Suarez R, Cerdeña J, Zumarán G, Alvarado M. 2021. Avispas parasitoides Ophioninae (Hymenoptera: Ichneumonidae) del departamento de Arequipa, Perú. Revista peruana de biología 28(2): e19387 (Mayo 2021). doi: http:// dx.doi.org/10.15381/rpb.v28i2.19387

$\begin{array}{ll}\text { Presentado: } & 01 / 01 / 2021 \\ \text { Aceptado: } & 09 / 05 / 2021 \\ \text { Publicado online: } & 25 / 05 / 2021 \\ \text { Editor: } & \text { Diana Silva }\end{array}$

\section{Resumen}

Se determinó la riqueza y composición de especies de avispas parasitoides nocturnas de la subfamilia Ophioninae en el departamento de Arequipa. Se evaluaron 16 localidades, a lo largo de un gradiente altitudinal que va desde el nivel del mar hasta los 4300 m; en cada localidad se realizaron colectas en los meses de marzo y mayo del 2018. Para la evaluación se emplearon trampas de luz. Se registraron 8 especies y 15 morfoespecies correspondientes a los géneros: Alophophion, Enicospilus, Eremotylus y Ophion. La mayor riqueza de Ophioninae se encontró a $3300 \mathrm{~m}(\mathrm{~S}=8)$. Los géneros Alophophion y Ophion fueron más abundantes en las localidades sobre los $2000 \mathrm{~m}$, Enicospilus fue más abundante en las localidades que se encontraban por debajo de los 1000 m, y Eremotylus solo se registró en una localidad a los 2300 m.

\section{Abstract}

The species richness and composition of nocturnal parasitoid wasps Ophioninae subfamily in the Arequipa department was determined. Sixteen localities were evaluated along an altitude gradient that goes from sea level up to $4300 \mathrm{~m}$; in each locality, collections were done in March and May 2018. Light traps were used for the evaluation. A total of 15 morphospecies and 8 species corresponding to the genera: Alophophion, Enicospilus, Eremotylus, and Ophion were registered. The highest richness of Ophioninae was found at $3300 \mathrm{~m} \mathrm{(S=8).} \mathrm{The} \mathrm{genera} \mathrm{Alopho-}$ phion and Ophion were more abundant at medium to high elevations, Enicospilus was more abundant at low altitude localities, and Eremotylus was only recorded in a medium altitude locality.

Palabras clave:

Enicospilus; Alophophion; avispas de Darwin; riqueza de especies.

Keywords:

Enicospilus; Alophophion; Darwin wasps; species richness.

\section{Introducción}

Las abejas, hormigas y avispas comprenden uno de los grupos más diversos del reino animal, el orden Hymenoptera (Fernández 2000). Este es uno de los cuatro órdenes megadiversos, con aproximadamente 150000 especies descritas y cerca de 85 familias (Somavilla et al. 2010). La Familia Ichneumonidae comprende la mayor diversidad de especies en el orden Hymenoptera, en esta se han descrito más de 25000 especies (Klopfstein et al. 2019) y más de 7400 de la Región Neotropical (Townes 1969, Quicke et al. 2009, Rodríguez-Berrío et al. 2009, Ruíz-Cancino 2015, García-Ramírez et al. 2016, Santos 2017). La fauna de Ichneumonidae ha sido poco estudiada en Perú, a pesar de ser una familia muy diversa (Rodríguez-Berrío \& Gutiérrez 2014). 
La subfamilia Ophioninae forma un grupo distintivo debido a su gran tamaño, apariencia esbelta y venación característica (Fernández-Triana 2005). Presenta 32 géneros y aproximadamente 1105 especies en todo el mundo (Lima et al. 2013, Sánchez et al. 2014, Shimizu \& Watanabe 2015, Alvarado 2016, Broad \& Shaw 2016, Suarez \& Alvarado 2020). La mayoría de Ophioninae son endoparásitos cenobiontes y solitarios, mayormente de larvas de Lepidoptera, varios son parasitoides comunes de plagas de lepidópteros en bosques y agro-ecosistemas (Gauld \& Lanfranco 1987, Gauld 1988, Sánchez et al. 2014, Quicke 2015). Debido a la facilidad de su captura con trampas de luz son apropiados para estudios zoogeográficos y ecológicos (Gauld \& Lanfranco 1987).

En el Perú hasta el momento, se han registrado 50 especies de Ophioninae distribuidas en nueve géneros (Rodríguez-Berrío et al. 2009, Alvarado 2011, Alvarado 2014, Sánchez et al. 2014, Alvarado 2016, Suarez \& Alvarado 2020). Mientras que para el departamento de Arequipa solo se tiene el registro de una especie recientemente descrita del género Eremotylus (Suarez \& Alvarado 2020). Por lo anterior, en este trabajo se brinda información sobre la riqueza y composición de especies de avispas parasitoides de la subfamilia Ophioninae en el departamento de Arequipa, basados en muestreos de 16 localidades distribuidas desde el nivel del mar hasta los $4300 \mathrm{~m}$ de altitud.

\section{Material y métodos}

Elárea de estudio comprende a las provincias de Arequipa: Camaná, Islay, Caravelí, Condesuyos, La Unión, Caylloma y Arequipa. Las evaluaciones se realizaron en 16 localidades a lo largo de una gradiente altitudinal desde el nivel del mar hasta los $4300 \mathrm{~m}$ de altitud, de las cuales cuatro localidades se ubicaron dentro de dos áreas naturales protegidas, la Reserva Paisajística Sub- cuenca del Cotahuasi y el Santuario Nacional Lagunas de Mejía (ver Tabla 1 y Fig. 1).

En cada localidad se realizaron dos colectas, durante los meses de marzo y mayo del 2018, en cada una durante tres noches consecutivas. Para la evaluación, en cada localidad, se instalaron dos trampas de luz por noche por cuatro horas consecutivas entre las 19:00 y 23:00 horas, como fuente de luz se utilizaron focos de luz mixta de 250 voltios y como fuente de energía generadores de electricidad portátiles (Sánchez et al. 2014). Los ejemplares recolectados fueron colocados en frascos que contenían alcohol al 95\%. y posteriormente llevados al laboratorio para su identificación.

Los especímenes recolectados fueron depositados en las colecciones del Museo de Historia Natural de la Universidad Nacional Mayor de San Marcos (MUSM, curador Gerardo Lamas), Museo de Historia Natural de la Universidad Nacional de San Agustín de Arequipa (MUSA, curador Evaristo Lopez) y la sección académica de Entomología y protección vegetal Escuela Profesional de Biología de la Universidad Nacional de San Agustín de Arequipa (curador Javier Huanca). Para la preparación de los ejemplares recolectados se realizó la técnica de montaje doble sugerido por Sarmiento (2006).

Para la identificación de la subfamilia se utilizaron las claves de Broad et al. (2018). Los géneros de Ophioninae se identificaron con las claves de Gauld y Lanfranco (1987). Para la determinación de las especies se emplearon claves para cada género: para Alophophion se utilizaron las claves propuestas por Alvarado (2014) y los grupos de especies sugeridos, para Enicospilus se utilizó la clave y grupos de especies propuestos por Gauld (1988) y para los géneros Eremotylus y Ophion se utilizó las claves de Gauld (1988). Todas las especies identificadas fueron comparadas con el material depositado en el MUSM.

Tabla 1. Localidades, coordenadas geográficas y altitudes de los puntos de muestreo.

\begin{tabular}{|c|c|c|c|c|c|}
\hline \multirow{2}{*}{ Provincia } & \multirow{2}{*}{ Distrito } & \multirow{2}{*}{ Localidad } & \multicolumn{2}{|c|}{ Coordenadas } & \multirow[t]{2}{*}{ Altitud (m) } \\
\hline & & & Longitud & Latitud & \\
\hline Camaná & Quilca & Quilca & -72.42164 & -16.71733 & 10 \\
\hline Islay & Mejía & Mejía(*) & -71.841607 & 17.165008 & 20 \\
\hline Camaná & Ocoña & Secocha & -72.57655 & -16.49867 & 160 \\
\hline Caravelí & Atiquipa & Atiquipa & -74.37721 & -15.76088 & 900 \\
\hline Condesuyos & Chuquibamba & Chuquibamba & -72.600803 & -15.87028 & 2000 \\
\hline La Unión & Cotahuasi & Sipia $(*)$ & -72.94945 & -15.23366 & 2100 \\
\hline Caylloma & Tapay & Tapay & -71.94806 & -15.59369 & 2300 \\
\hline Arequipa & Pocsi & Pocsi & -71.43775 & 16.505701 & 2400 \\
\hline Arequipa & Yura & Yura & -71.706249 & -16.22711 & 2800 \\
\hline Caylloma & Maca & Maca & -71.79297 & -15.63081 & 3100 \\
\hline La Unión & Cotahuasi & Puyca(*) & -72.72233 & -15.06807 & 3150 \\
\hline Condesuyos & Chuquibamba & Chuquibamba & -72.67025 & -15.80924 & 3300 \\
\hline Condesuyos & Chuquibamba & Chuquibamba & -72.68693 & -15.77832 & 3800 \\
\hline La Unión & Cotahuasi & Cotahuasi $(*)$ & -72.89017 & -15.25857 & 3900 \\
\hline Arequipa & Chiguata & Chiguata & -71.319202 & -16.39268 & 4000 \\
\hline Arequipa & San Juan de Tarucani & Tocra & -71.46233 & -15.84635 & 4300 \\
\hline
\end{tabular}

* Localidades en áreas naturales protegidas 


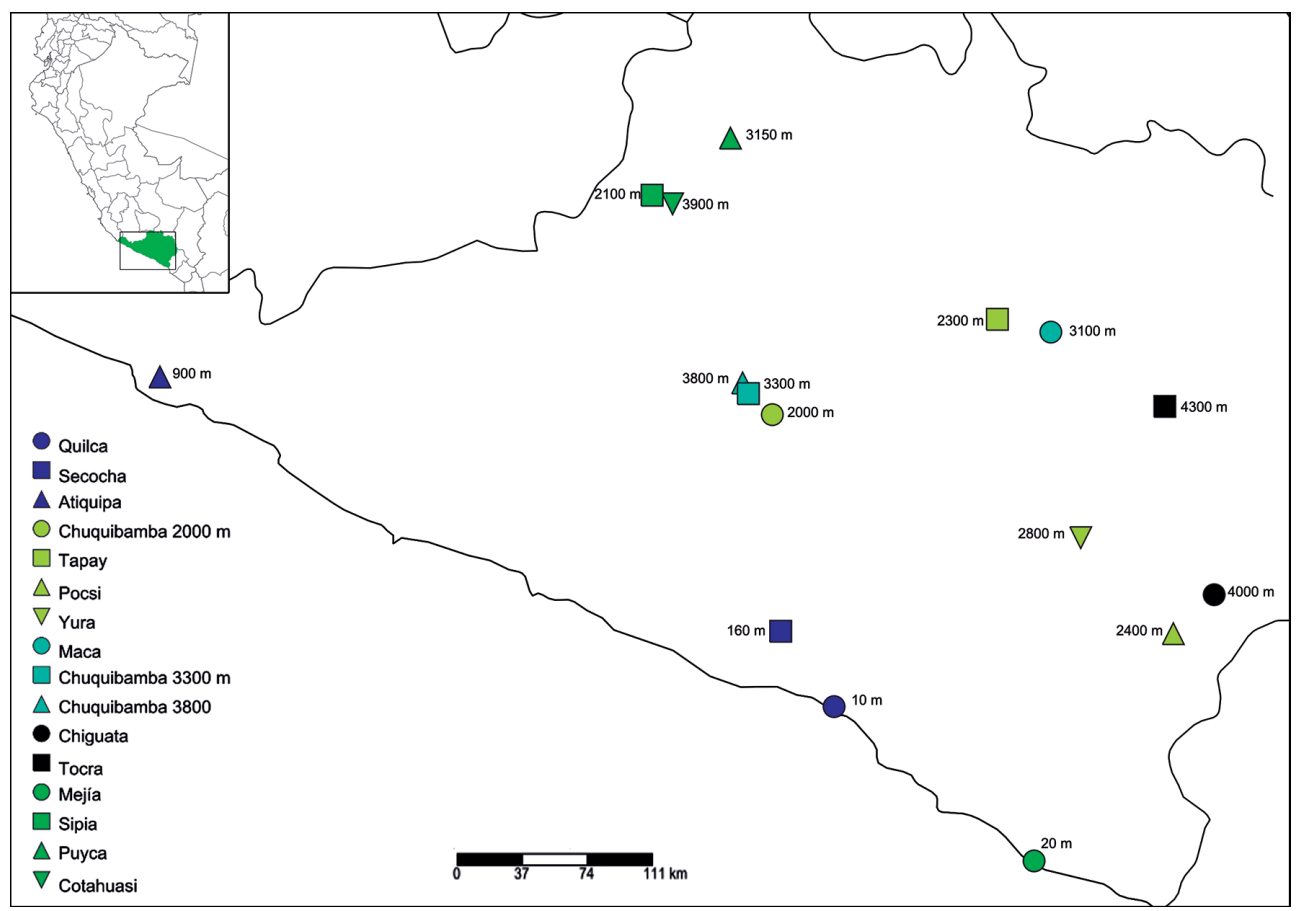

Figura 1. Mapa de Arequipa con la distribución de las localidades evaluadas. Localidades en color verde están en áreas naturales protegidas.

Las fotografías del hábito se tomaron con una cámara digital Canon T3I conectada a una lente microscópica de larga distancia Infinity K-2 y para la toma de fotografías de estructuras más pequeñas como la vista lateral y frontal de la cabeza, la venación alar y el propodeo se utilizó un estereoscopio con cámara ZEISS Axiocam ERc 5s. Las fotos digitales se combinaron utilizando el programa Zerene. Las láminas se prepararon utilizando Adobe Photoshop CS6. Se utilizó el programa Microsoft Excel para la elaboración de matrices de datos que dieron lugar a las tablas y gráficas.

\section{Resultados y discusión}

Se recolectaron un total de 255 especímenes de la subfamilia Ophioninae, de los cuales 201 pertenecían al género Alophophion, 47 a Ophion, seis a Enicospilus y uno a Eremotylus (Tabla 2). Se encontraron 185 hembras, 68 machos y dos especímenes indeterminados (carecían de la parte terminal del abdomen). Se registraron un total de nueve especies y 14 morfoespecies, las que serán referidas en el resto del texto como especies (Fig. 2).

La localidad con mayor abundancia de Ophioninae fue Puyca (3150 m) con 93 especímenes y las localidades con menor abundancia fueron Tapay $(2300 \mathrm{~m})$ y Cotahuasi (3900 m) con un solo espécimen en cada localidad (Tabla 3). Alophophion y Ophion fueron más abundantes en marzo, Eremotylus solo se recolectó en marzo y Enicospilus fue más abundante en mayo. En marzo se recolectó mayor cantidad de especímenes en comparación con el muestreo de mayo, esto se explicaría principalmente por la mayor cobertura vegetal y lluvias en marzo, a diferencia de mayo cuando la cobertura vegetal había desaparecido y las zonas se observaban secas, por lo tanto, podemos esperar que mayor cobertura de alimento para los hospederos incrementaría el número de parasitoides en la zona (Shaw 2006).

Las localidades con menor riqueza de especies fueron Cotahuasi (3900 m), donde se registró solo a E. flavoscutellatus, Mejía ( $20 \mathrm{~m}$ ) donde se registró solo a O. flavidus, y Tapay $(2300 \mathrm{~m})$ donde se registró a Eremotylus pukaya$n a$. La localidad con mayor riqueza de especies fue Chuquibamba $(3300 \mathrm{~m})$ donde se registraron ocho especies en total, siete correspondientes al género Alophophion y una especie de Ophion, seguido de Puyca (3150 m) con siete especies en total, cinco de Alophophion y dos de Ophion. Solo en Secocha $(160 \mathrm{~m})$ se registró la ocurrencia de tres géneros: Alophophion, Enicospilus y Ophion, no hubo ninguna localidad que registrara la ocurrencia de los cuatro géneros (Tabla 3). Las localidades donde no se colectaron Ophioninae fueron Yura (2800 m) y Tocra $(4300 \mathrm{~m})$, posiblemente relacionado a las condiciones climáticas como los fuertes vientos y la baja temperatura durante el muestreo.

El género que presenta mayor riqueza es Alophophion con 15 especies, seguido de Ophion con cuatro especies, Enicospilus con tres especies y Eremotylus con solo una especie. En el muestreo realizado en marzo se registraron 18 especies, mientras que en mayo solo se recolectaron ocho especies (Tabla 3). La diferencia en el número de especies entre estos dos meses podría sugerir estacionalidad, pero para poder explicar esto sería necesario un mayor número de muestreos al año. Sin embargo, se conoce que algunas especies de Enicospilus exhiben una marcada estacionalidad, pasando todo el año en diapausa dentro de la pupa de su hospedero, y solo salen cuando inicia la época de lluvias (Hanson \& Gauld 2006). 


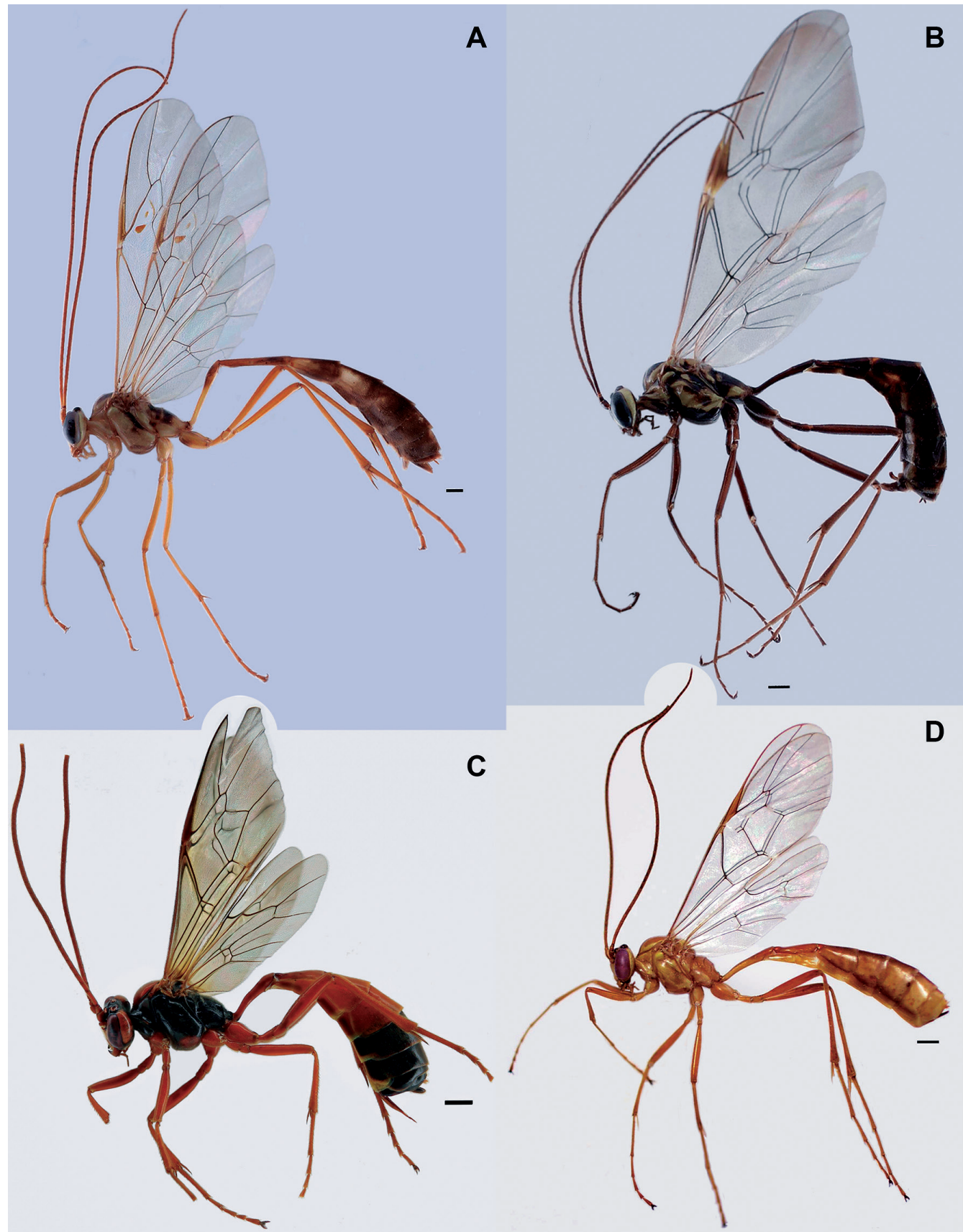

Figura 2. Géneros registrados en el departamento de Arequipa. Hábitus, vista lateral. (A) Enicospilus flavoscutellatus, (B) Alophophion capacyupanquii, (C) Eremotylus pukayana y (D) Ophion flavidus.

El género más abundante en el presente estudio fue Alophophion, este género ha sido también encontrado en ambientes áridos y/o fríos (Alvarado 2014), lo cual sería semejantes a los ambientes áridos de Arequipa donde fue encontrado este. Sin embargo en Perú, Sánchez et al. (2014) en ambientes como el bosque nublado montano y pajonal reportaron abundante a Enicospilus, lo cual concuerda con lo encontrado por Gauld (1988) en su evaluación de bosques húmedos tropicales. Las diferencias de hábitat también explicarían la presencia de Eremotylus en Arequipa, género que suele habitar ambientes semiáridos (Gauld 1988).

En cuanto a la ocurrencia de los géneros respecto a la altura, Alophophion fue recolectado entre los $10 \mathrm{y}$
$4000 \mathrm{~m}$, pero con mayor abundancia en la localidad de Puyca (3150 m); Enicospilus fue registrado entre los 10 y 900 m y también a los 3900 m, pero fue más abundante en Secocha $(160 \mathrm{~m})$; Eremotylus solo fue recolectado en Tapay $(2300 \mathrm{~m})$ y Ophion fue recolectado entre los 20 y 3800 m, pero fue mucho más abundante en Pocsi (2400 m) (Fig. 3).

La especie más abundante fue A. chavinaensis, con 77 especímenes. Mientras que el género más raro fue Eremotylus, representado por solo una especie, E. pukayana, con un solo ejemplar hembra recolectada en la localidad de Tapay (2300 m). La especie más abundante de Ophion fue Ophion sp. 3 con 20 especímenes y la especie de menor abundancia fue Ophion sp. 1 con siete especímenes. 
Tabla 2. Lista de especímenes hembras, machos e indeterminados de las especies de la subfamilia Ophioninae

\begin{tabular}{|c|c|c|c|c|}
\hline Taxa & Hembras & Machos & Ind. & Total \\
\hline Alophophion & 152 & 48 & 1 & 201 \\
\hline Alophophion capacyupanquii Alvarado, 2014 & 0 & 1 & 0 & 1 \\
\hline Alophophion chavinaensis Alvarado, 2014 & 69 & 8 & 0 & 77 \\
\hline Alophophion huascari Alvarado, 2014 & 8 & 1 & 0 & 9 \\
\hline Alophophion maytacapaci Alvarado, 2014 & 0 & 1 & 0 & 1 \\
\hline Alophophion yupankii Alvarado, 2014 & 6 & 2 & 1 & 9 \\
\hline Alophophion sp. 1 & 3 & 1 & 0 & 4 \\
\hline Alophophion sp. 2 & 1 & 2 & 0 & 3 \\
\hline Alophophion sp. 3 & 17 & 0 & 0 & 17 \\
\hline Alophophion sp. 4 & 13 & 1 & 0 & 14 \\
\hline Alophophion sp. 5 & 8 & 14 & 0 & 22 \\
\hline Alophophion sp. 6 & 0 & 1 & 0 & 1 \\
\hline Alophophion sp. 7 & 2 & 10 & 0 & 12 \\
\hline Alophophion sp. 8 & 15 & 0 & 0 & 15 \\
\hline Alophophion sp. 9 & 0 & 1 & 0 & 1 \\
\hline Alophophion sp. 10 & 10 & 5 & 0 & 15 \\
\hline Enicospilus & 3 & 3 & 0 & 6 \\
\hline Enicospilus flavoscutellatus Brullé, 1846 & 0 & 1 & 0 & 1 \\
\hline Enicospilus purgatus Say, 1835 & 0 & 1 & 0 & 1 \\
\hline Enicospilus sp. 1 & 3 & 1 & 0 & 4 \\
\hline Eremotylus & 1 & 0 & 0 & 1 \\
\hline Eremotylus pukayana Suarez y Alvarado, 2020 & 1 & 0 & 0 & 1 \\
\hline Ophion & 29 & 17 & 1 & 47 \\
\hline Ophion flavidus Brullé, 1846 & 8 & 2 & 0 & 10 \\
\hline Ophion sp. 1 & 6 & 1 & 0 & 7 \\
\hline Ophion sp. 2 & 6 & 4 & 0 & 10 \\
\hline Ophion sp. 3 & 9 & 10 & 1 & 20 \\
\hline TOTAL & 185 & 68 & 2 & 255 \\
\hline
\end{tabular}

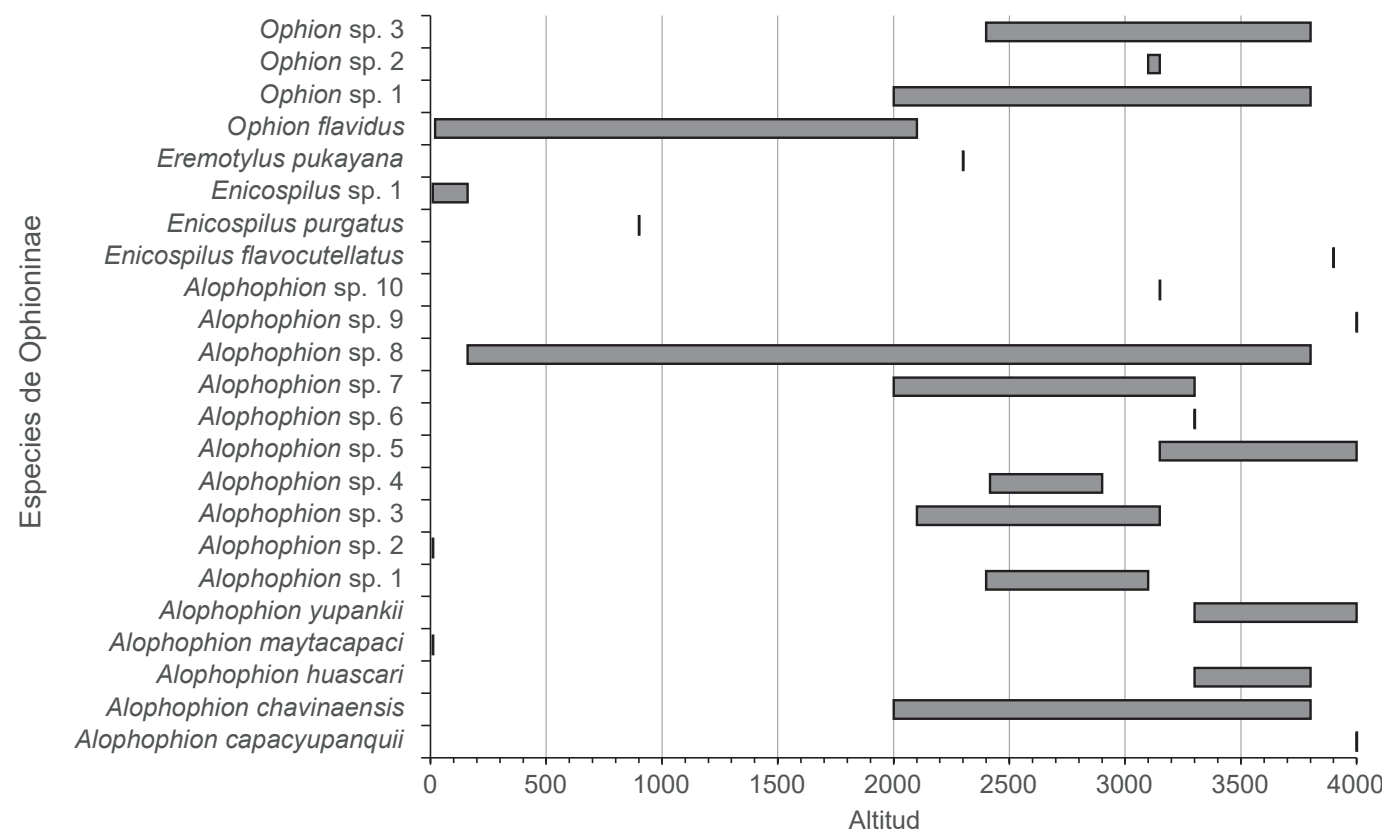

Figura 3. Distribución altitudinal de las especies y morfoespecies de la subfamilia Ophioninae. 
Tabla 3. Riqueza y abundancia de especies de la subfamilia Ophioninae evaluadas en el departamento de Arequipa por localidad. $\mathrm{s}=$ número de especies; $\mathrm{n}=$ número de individuos en los meses de marzo y mayo.

\begin{tabular}{|c|c|c|c|c|c|c|c|c|c|c|c|c|c|c|}
\hline & \multicolumn{4}{|c|}{ Alophophion } & \multicolumn{4}{|c|}{ Enicospilus } & \multirow{2}{*}{\multicolumn{2}{|c|}{$\begin{array}{c}\text { Eremotylus } \\
\text { Marzo }\end{array}$}} & \multicolumn{4}{|c|}{ Ophion } \\
\hline & \multicolumn{2}{|c|}{ Marzo } & \multicolumn{2}{|c|}{ Mayo } & \multicolumn{2}{|c|}{ Marzo } & \multicolumn{2}{|c|}{ Mayo } & & & \multicolumn{2}{|c|}{ Marzo } & \multicolumn{2}{|c|}{ Mayo } \\
\hline & $\mathrm{n}$ & $\mathbf{s}$ & $\mathrm{n}$ & $s$ & $\mathrm{n}$ & 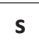 & $n$ & $s$ & $n$ & $s$ & $n$ & $s$ & $n$ & $s$ \\
\hline Quilca (10) & 0 & 0 & 4 & 2 & 0 & 0 & 1 & 1 & 0 & 0 & 0 & 0 & 0 & 0 \\
\hline Mejía (20) & 0 & 0 & 0 & 0 & 0 & 0 & 0 & 0 & 0 & 0 & 5 & 1 & 0 & 0 \\
\hline Secocha (160) & 1 & 1 & 0 & 0 & 0 & 0 & 3 & 1 & 0 & 0 & 0 & 0 & 3 & 1 \\
\hline Atiquipa (900) & 0 & 0 & 0 & 0 & 1 & 1 & 0 & 0 & 0 & 0 & 1 & 1 & 0 & 0 \\
\hline Chuquibamba (2000) & 7 & 1 & 2 & 1 & 0 & 0 & 0 & 0 & 0 & 0 & 2 & 1 & 0 & 0 \\
\hline Sipia (2100) & 1 & 1 & 0 & 0 & 0 & 0 & 0 & 0 & 0 & 0 & 1 & 1 & 0 & 0 \\
\hline Tapay (2300) & 0 & 0 & 0 & 0 & 0 & 0 & 0 & 0 & 1 & 1 & 0 & 0 & 0 & 0 \\
\hline Pocsi (2400) & 4 & 2 & 0 & 0 & 0 & 0 & 0 & 0 & 0 & 0 & 19 & 1 & 0 & 0 \\
\hline Yura (2800) & 0 & 0 & 0 & 0 & 0 & 0 & 0 & 0 & 0 & 0 & 0 & 0 & 0 & 0 \\
\hline Maca (3100) & 2 & 2 & 0 & 0 & 0 & 0 & 0 & 0 & 0 & 0 & 2 & 2 & 0 & 0 \\
\hline Puyca (3150) & 53 & 5 & 25 & 1 & 0 & 0 & 0 & 0 & 0 & 0 & 3 & 2 & 8 & 1 \\
\hline Chuquibamba (3300) & 45 & 6 & 5 & 2 & 0 & 0 & 0 & 0 & 0 & 0 & 1 & 1 & 0 & 0 \\
\hline Chuquibamba (3800) & 18 & 3 & 7 & 1 & 0 & 0 & 0 & 0 & 0 & 0 & 2 & 2 & 0 & 0 \\
\hline Cotahuasi (3900) & 0 & 0 & 0 & 0 & 1 & 1 & 0 & 0 & 0 & 0 & 0 & 0 & 0 & 0 \\
\hline Chiguata (4000) & 13 & 4 & 0 & 0 & 0 & 0 & 0 & 0 & 0 & 0 & 0 & 0 & 0 & 0 \\
\hline Tocra (4300) & 0 & 0 & 0 & 0 & 0 & 0 & 0 & 0 & 0 & 0 & 0 & 0 & 0 & 0 \\
\hline Total & 144 & & 43 & & 2 & & 4 & & 1 & & 36 & & 11 & \\
\hline
\end{tabular}

Alophophion presentó la mayor riqueza de especies en la localidad de Chuquibamba (3300 m) y la menor riqueza de especies en Secocha (160 m) y Sipia (2100 m) con una especie en cada localidad respectivamente. Enicospilus presentó una sola especie en las localidades de Quilca (10 m), Secocha (160 m), Atiquipa (900 m) y Cotahuasi (3900 m). El género Ophion presentó la mayor riqueza de especies en las localidades de Maca (3100 m), Puyca (3150 m) y Chuquibamba (3800 m) con dos especies en cada localidad respectivamente, y la menor riqueza la presentó en seis localidades las cuales solo registran una especie cada una (Tabla 3). Nuestros resultados coinciden con lo presentado por Sánchez et al. (2014) que afirman que Alophophion es predominante a mediana y elevada altitud (sobre los 2000 m), Enicospilus de baja a mediana altitud y Ophion a mediana y elevada altitud.

La composición de especies a lo largo de una gradiente altitudinal en ambientes áridos y en ambientes húmedos de Perú es diferente. En nuestro trabajo, Alophophion es el género más rico en especies y abundancia, encontrado a lo largo de la gradiente y no restringido sobre los 2000 m como lo encuentró Sánchez et al. (2014) en el bosque nublado montano y pajonal. Enicospilus flavoscutellatus y E. purgatus están ampliamente distribuidas en el continente americano (Gauld 1988), pero en nuestro estudio están representadas por un individuo cada uno; lo que podría sugerir que este género sería raro en ambientes áridos.

Sin embargo, al igual que en el estudio de Sánchez et al. (2014), en nuestro trabajo Ophion también es comúnmente encontrado sobre los 2000 m, con la excepción de
O. flavidus que fue encontrada por debajo de los $2000 \mathrm{~m}$. Ophion flavidus se encuentra ampliamente distribuido en el continente americano y comúnmente se encuentra en ambientes disturbados y agrícolas (Gauld 1988).

\section{Literatura citada}

Alvarado M. 2011. Biodiversidad de Ichneumonidae (Hymenoptera) en tres hábitats de ecosistemas de Costa, Sierra y Selva de Perú. Tesis para optar el grado de Magister Scientitae en Entomología. Universidad Nacional Agraria La Molina, Lima, Perú.

Alvarado M. 2014. Revision of the South American wasp genus Alophophion Cushman, 1947 (Hymenoptera: Ichneumonidae: Ophioninae). Revista Peruana de Biología 21(1):03-60. https://doi.org/10.15381/rpb. v21i1.8245

Alvarado M. 2016. A remarkable new species of Sicophion Gauld, 1979 (Hymenoptera: Ichneumonidae) from Peru, with a key to the species. Zootaxa 4138(1):195200. https://doi.org/10.11646/zootaxa.4138.1.11

Broad G.R., Shaw M.R. \& Fitton M.G. 2018. Handbooks for the Identification of British Insects: Ichneumonid Wasps (Hymenoptera: Ichneumonidae): Their Classification and Biology. Royal Entomological Society, 7(12): vi + 1-418.

Broad G. \& R. Shaw. 2016. The British species of Enicospilus (Hymenoptera: Ichneumonidae: Ophioninae). European Journal of Taxonomy 187: 1-31. https://doi. org/10.5852/ejt.2016.187

Fernández F. 2000. Sistemática y filogenia de los himenópteros de la región Neotropical: Estado del conocimiento y perspectivas. F. Martínez-Piera, J. J. Morrone y A. Melic (Eds.), Hacia un proyecto CYTED para el inventario y estimación de la diversidad entomológica en Iberoamérica 1:211-231. 
Fernández-Triana J. 2005. The taxonomy and biogeography of Cuban Ophioninae (Hymenoptera: Ichneumonidae). Zootaxa 1007(1):1-60. https://doi.org/10.11646/ zootaxa.1007.1.1

García-Ramírez J, Ruíz-Cancino E, Coronado-Blanco M, Khalaim I. 2016. Ichneumonidae (Hymenoptera: Ichneumonoidea) de Escárcega, Campeche, México: nuevos registros de especies. CienciaUAT 10(2):6-12. https://doi. org/10.29059/cienciauat.v10i2.544

Gauld D, Lanfranco D. 1987. Los géneros de Ophioninae de Centro y Sudamérica. Revista de Biología Tropical 35(2):257-267.

Gauld D. 1988. A survey of the Ophioninae (Hymenoptera: Ichneumonidae) of tropical Mesoamerica with special reference to the fauna of Costa Rica. Bulletin of the British Museum Natural History. Entomology Series 57(1):74-301.

Hanson P, Gauld D. 2006. Hymenoptera de la región Neotropical. Memoirs of the American Entomological Institute 77:446-454.

Klopfstein S, Santos BF, Shaw MR, Alvarado M, Bennett AMR, Pos DD, Giannotta M, Florez AFH, Karlsson D, Khalaim AI, et al. 2019. Darwin wasps: a new name heralds renewed efforts to unravel the evolutionary history of Ichneumonidae. Entomological Communications 1:ec01006-ec01006. http://dx.doi. org/10.37486/2675-1305.ec01006

Lima R, Jacobi M, Kumagai F. 2013. Review of the neotropical species of Stauropoctonus Brauns, 1889 (Hymenoptera: Ichneumonidae: Ophioninae). Zootaxa 3750(5):494-514. https://doi.org/10.11646/zootaхa.3750.5.4

Quicke L, Laurenne M, Fitton G, Broad R. 2009. A thousand and one wasps: a $28 \mathrm{~S}$ rDNA and morphological phylogeny of the Ichneumonidae (Insecta: Hymenoptera) with an investigation into alignment parameter space and elision. Journal of Natural History 43(23-24):1305-1421. https://doi.org/10.1080/00222930902807783

Quicke L. 2015. The braconid and ichneumonid parasitoid wasps: biology, systematics, evolution and ecology. Chennai, India: John Wiley \& Sons. 752pp. https://doi. org/10.1002/9781118907085

Rodríguez-Berrío A, Bordera S, Sääksjärvi E. 2009. Checklist of Peruvian Ichneumonidae (Insecta, Hymenoptera). Zootaxa 2303:1-44. https://doi.org/10.11646/zootaха.2303.1.1

Rodríguez-Berrío A, Gutiérrez B. 2014. Diversidad de la subfamilia Campopleginae-Ichneumonidae (Hymenoptera) en la cuenca del Río Cañete-Lunahuaná, Perú. Ecología Aplicada 13(2):147-152. https://doi.org/10.21704/ rea.v13i1-2.465

Ruíz-Cancino E. 2015. La familia Ichneumonidae (Hymenoptera) en México. Entomología mexicana, 2(1), 1-13.

Sánchez E, Alvarado M, Grados J. 2014. Comunidad de avispas Ophioninae (Hymenoptera: Ichneumonidae) en el bosque nublado Monteseco, Cajamarca, Perú. Revista Peruana de Biología 21(3): 229-234. https://doi. org/10.15381/rpb.v21i3.10896

Santos F. 2017. Phylogeny and reclassification of Cryptini (Hymenoptera, Ichneumonidae, Cryptinae), with implications for ichneumonid higher-level classification. Systematic Entomology 42(4):650-676. https://doi. org/10.1111/syen.12238
Sarmiento C. 2006. Métodos generales de recolección. En Fernández F. y M. Sharkey. Introducción a los Hymenoptera de la Región Neotropical (pp.115-131). Bogotá: Guadalupe Ltda.

Shaw M. 2006. Habitat considerations for parasitic wasps (Hymenoptera). Journal of Insect Conservation 10:117 127. https://doi.org/10.1007/s10841-006-6288-1

Shimizu S, Watanabe K. 2015. Discovery of the genus Leptophion Cameron, 1901, from Japan and the Palaearctic region, with description of two new species (Hymenoptera: Ichneumonidae: Ophioninae). Zootaxa 4000(1):111-122. https://doi.org/10.11646/zootaxa.4000.1.5

Somavilla A, Köhler A, Hermes G. 2010. Contribuição aos estudos dos Vespidae ocorrentes no estado do Rio Grande do Sul (Insecta, Hymenoptera). Revista Brasileira de Biociências 8(3):257-263.

Suarez R, Alvarado M. 2020. A new species of Eremotylus Forster, 1869 (Hymenoptera: Ichneumonidae) from Peru, with a key to the Neotropical species. Zootaxa 4758(2):397-400. https://doi.org/10.11646/zootaха. 4758.2 .13

Townes H. 1969. The genera of Ichneumonidae, part 1. Anzeiger für Schädlingskunde 42(7):110-110. https://doi. org/10.1007/BF02027741

\section{Agradecimientos / Acknowledgments:}

Agradecemos al equipo del Museo de Historia Natural de la UNSA que estuvo conformado por: L. Olivera, S. Ramos, M. Arivilca, M. Condori, S. Chambi., E. Olanda, H. Quispe, A. Aspur, M. Mojorovich, C. Palacios, A. Quispe, K. Rosas, Y. Aguilar, L. Pinto, G. Aliaga y G. Rodriguez por ayudarnos a realizar los muestreos; a los docentes Cesar Luque, Luis Villegas y Javier Huanca de la Escuela de Biología de la UNSA por su apoyo durante la investigación; a la Sección Académica de Entomología y Protección Vegetal de la Escuela de Biología de la UNSA y al Departamento de Entomología del Museo de Historia Natural de la UNMSM por proporcionarnos los equipos que fueron necesarios para llevar a cabo esta investigación. Los permisos de colecta fueron emitidos por SERFOR (R.A. No 0024-2018-SERFOR-ATFFS AREQUIPA-ADC) y SERNANP (No 288-2018-SERNANP-DGANP).

Conflicto de intereses / Competing interests:

Los autores declaran que no incurren en conflictos de intereses.

\section{Rol de los autores / Authors Roles:}

RS: Conceptualización, Investigación, Redacción: revisión y edición. JC: Recursos, Investigación, Redacción: revisión y edición. GZ: Recursos, Redacción: revisión y edición. MA: Conceptualización, Investigación, Redacción: revisión y edición, Validación.

\section{Fuentes de financiamiento / Funding:}

Los autores declaran que este trabajo no recibió financiación específica.

\section{Aspectos éticos / legales; Ethics / legals:}

Los autores declaran no haber incurrido en aspectos antiéticos. Las colectas se realizaron bajo los permisos de colecta otorgados por SERFOR (R.A. No 0024-2018-SERFOR-ATFFS-AREQUIPA-ADC) y SERNANP (No 288-2018-SERNANP-DGANP). 
Página en banco

Blank page 